\title{
Abundance patterns in the low-metallicity emission-line galaxies from the Early Data Release of the Sloan Digital Sky Survey
}

\author{
Y. I. Izotov ${ }^{1}$, G. Stasińska ${ }^{2}$, N. G. Guseva ${ }^{1}$, and T. X. Thuan ${ }^{3}$ \\ 1 Main Astronomical Observatory, Ukrainian National Academy of Sciences, Zabolotnoho 27, Kyiv 03680, Ukraine \\ ${ }^{2}$ LUTH, Observatoire de Meudon, 92195 Meudon Cedex, France \\ 3 Astronomy Department, University of Virginia, Charlottesville, VA 22903, USA
}

Received 23 July 2003 / Accepted 3 November 2003

\begin{abstract}
We have derived element abundances in 310 emission-line galaxies from the Early Data Release of the Sloan Digital Sky Survey (SDSS) for which the [O III] $\lambda 4363$ emission line was detected, allowing abundance determination by direct methods. We found no extremely metal-deficient galaxy $\left(Z \lesssim Z_{\odot} / 12\right)^{\star}$, probably as a consequence of selection effects in the SDSS sample. The oxygen abundance $12+\log \mathrm{O} / \mathrm{H}$ of the SDSS galaxies sample lies in the range from $\sim 7.6\left(Z_{\odot} / 12\right)$ to $\sim 8.4\left(Z_{\odot} / 2\right)$. This sample is merged with a sample of $\sim 100$ blue compact dwarf galaxies with high quality spectra containing some very low-metallicity objects to study the abundance patterns of low-metallicity emission-line galaxies. We find that the $\alpha$ elementto-oxygen abundance ratios do not show any significant trends with the oxygen abundance, in agreement with previous studies. $\mathrm{The} \mathrm{Fe} / \mathrm{O}$ abundance ratio is smaller than the solar value, which we interpret as an indication that type Ia supernovae have not yet appeared in these galaxies, implying an age of less than 1-2 Gyr. However, a slight decrease of the Fe/O abundance ratio with increasing metallicity suggests some depletion of iron onto dust in the galaxies with higher metallicities. The N/O abundance ratio ranges from $\log \mathrm{N} / \mathrm{O}=-1.6$ to -0.8 . The fact that no galaxy with $\log \mathrm{N} / \mathrm{O} \lesssim-1.6$ was discovered implies that local low-metallicity emission-line galaxies are of a different nature than high-redshift damped Ly $\alpha$ systems with $\log$ N/O of $\sim-2.3$ and that their ages are probably larger than 100-300 Myr. Our data indicate the existence of a gradual nitrogen enrichment on a time-scale of a few Myr.
\end{abstract}

Key words. galaxies: fundamental parameters - galaxies: starburst - galaxies: abundances

\section{Introduction}

The study of element abundances in low-metallicity emissionline galaxies is important for our understanding of the chemical evolution of galaxies and for constraining models of stellar nucleosynthesis and the shape of the initial mass function. The optical spectra of H II regions in these galaxies show strong narrow emission lines superposed on a stellar continuum that is rising toward the blue, allowing abundance determinations of such heavy elements as nitrogen, oxygen, neon, sulfur, argon, and iron.

The oxygen abundance in the most metal-poor emissionline galaxies is around 1/30 times the solar value, which makes these objects among the least chemically evolved galaxies in the universe. It was proposed (Sargent \& Searle 1970) that the most metal-poor galaxies were actually experiencing their first burst of star formation. However, subsequent photometric and spectroscopic studies have shown that the majority of metal-poor galaxies also possess several Gyr old stellar populations. In the case of the two extremely metal-deficient galaxies,

Send offprint requests to: Y. I. Izotov, e-mail: izotov@mao.kiev.ua $\star 12+\log (\mathrm{O} / \mathrm{H})_{\odot}=8.69 \pm 0.05($ Lodders 2003$)$.
I Zw 18 and SBS 0335-052, there is however no clear evidence of such stellar population and these galaxies may be genuinely young (e.g., Hunt et al. 2003; Guseva et al. 2003c).

Studies of local metal-poor emission-line galaxies are also crucial in the context of galaxy formation. The proximity of these metal-poor galaxies allows studies of their structure, metal content, and stellar populations in a nearly pristine environment with a sensitivity, precision, and spatial resolution that faint, small angular size, distant high-redshift galaxies do not allow. In particular, the comparison of the abundance patterns in the local low-metallicity dwarf galaxies and distant damped Ly $\alpha$ systems (DLA) can shed light on the properties and the evolution of the primeval high-redshift galaxies.

The abundances of CNO play an especially important role in the understanding the chemical evolution of the galaxies. It is well established (e.g., Maeder 1992) that oxygen and likely carbon are produced mainly by massive stars. The origin of nitrogen is more controversial.

In local metal-poor star-forming galaxies the nitrogen-tooxygen abundance ratio is found to be constant (e.g., Garnett 1990) with a very low dispersion around the mean value of $\log \mathrm{N} / \mathrm{O}=-1.6$ for galaxies with oxygen abundance 
$12+\log \mathrm{O} / \mathrm{H} \lesssim 7.6$ (Thuan et al. 1995; Izotov \& Thuan 1999). This led Thuan et al. (1995) and Izotov \& Thuan (1999) to infer that in those galaxies, nitrogen has been produced by massive stars as a primary element and hence the lowest-metallicity galaxies are young systems. However, Henry et al. (2000) proposed another explanation involving a star formation occuring at a very low rate, with massive stars producing oxygen and intermediate-mass stars producing primary nitrogen with a typical time-lag of $250 \mathrm{Myr}$.

In high-redshift low-metallicity damped Ly $\alpha$ systems, on the other hand, the spread of the N/O abundance ratio appears to be very large (e.g., Lu et al. 1998; Centurión et al. 2003). The N/O abundance ratio in some DLAs is found to be one order of magnitude lower than that in blue compact dwarf galaxies (BCD) of same metallicity. If real, such differences suggest that DLAs and BCDs are systems in different evolutionary stages. This also raises doubts on the young age of the lowestmetallicity BCDs and suggests that the production of nitrogen by intermediate-mass stars is actually important in the lowmetallicity BCDs and DLAs. This is in line with predictions of new models of rotating stars (e.g., Meynet \& Maeder 2002), although these models also predict the production of a large amount of primary nitrogen by low-metallicity massive stars.

Emission-line galaxies with very low metallicity are rare. Only two dozens of BCDs with an oxygen abundance $12+$ $\log \mathrm{O} / \mathrm{H} \lesssim 7.6$ are known up to now. The aim of this paper is to produce a large sample of low-metallicity emission-line galaxies with reliably derived element abundances and to compare them with those in the high-redshift DLAs. This should allow a better understanding of the nitrogen production and of the evolutionary status of both types of objects. We have used the early data release (EDR) of the Sloan Digital Sky Survey (SDSS) (Stoughton et al. 2002) which includes the spectra of $\sim 50000$ galaxies, and searched for low-metallicity emission-line galaxies. The procedure and the resulting sample are described in Sect. 2. The derivation of the elemental abundances is presented and discussed in Sect. 3. The main conclusions of this study are presented in Sect. 4.

\section{The sample}

The flux-calibrated spectra of the SDSS galaxies from the EDR have been extracted from the Space Telescope Science Institute archives $^{1}$.

From an examination of $\sim 50000$ spectra, we selected a sample of 310 emission-line galaxies with an emission feature at the wavelength of the [O III] $\lambda 4363$ emission line detected at least at the $1 \sigma$ level. The presence of this line allows a direct (as opposed to statistical or model-dependent) determination of the electron temperature and abundances. It also discriminates against metal-rich galaxies, in which important cooling of the $\mathrm{H}$ II regions by metals strongly reduces the excitation of this line. Because the wavelength scale of the extracted spectra is nonlinear they have been transformed to a linear wavelength scale. Then spectra have been reduced to a zero redshift using the galaxy redshifts provided in the EDR. Typical

\footnotetext{
${ }^{1}$ http://archive.stsci.edu/sdss
}

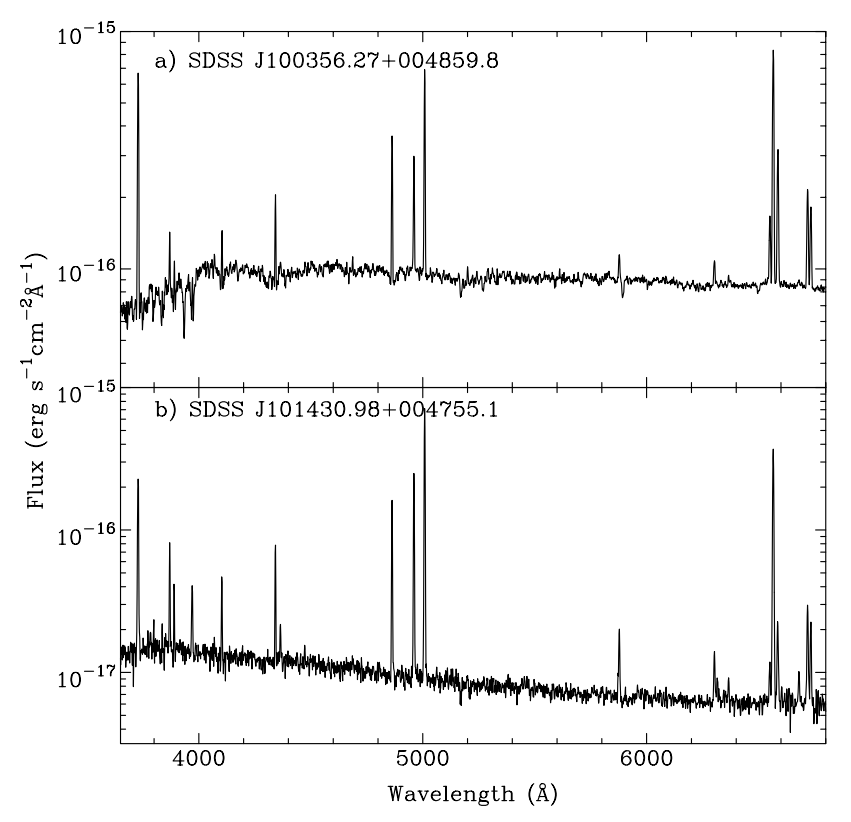

Fig. 1. Typical spectra of the a) low-excitation and b) high-excitation $\mathrm{H}$ II regions from the SDSS EDR. The spectra are transformed to a linear wavelength scale and zero redshift.

spectra of the galaxies from the SDSS sample are shown in Fig. 1. The upper spectrum is of lower excitation as compared to the lower spectrum as evidenced by the fluxes of [O II] $\lambda 3727$ and [O III] $\lambda 4959,5007$ emission lines relative to $\mathrm{H} \beta$. Emission line fluxes have been measured using Gaussian fitting. They have been corrected for interstellar extinction using the observed hydrogen Balmer line fluxes. The spectra in the SDSS sample cover the wavelength range $\sim \lambda 3820-9300 \AA$, therefore the line [O II] $\lambda 3727$ has not been detected in lowredshift galaxies with $z \lessgtr 0.025$. In those cases we used the [O II] $\lambda 7320,7331$ emission lines. Another problem with the SDSS spectra is that in many cases some of the strongest lines, most often $\mathrm{H} \alpha$ and $[\mathrm{O}$ III] $\lambda 5007$, are clipped. Therefore, many objects with strong emission lines were not included in our sample. However, in the cases when only $\mathrm{H} \alpha$ and [O III] $\lambda 5007$ emission lines are clipped, their fluxes are adopted to be respectively $2.8 \times I(\mathrm{H} \beta)$ and $3 \times I([\mathrm{O} \mathrm{III}] \lambda 4959)$.

Figure 2 shows the classical diagnostic diagram [O III] $\lambda 5007 / \mathrm{H} \beta$ vs. [N II] $\lambda 6583 / \mathrm{H} \alpha$ used to distinguish objects ionized by massive main sequence stars from objects ionized by non-thermal radiation. The dashed line separates the former (labeled "H II") from the latter (labeled "AGN") (Osterbrock 1989). Most of our selected objects fall in the "H II" region on the diagram. Only in two galaxies the ionization by a non-thermal radiation is important. Note that the situation is very different when considering an entire sample of emission-line galaxies without imposing the detection of [O III] $\lambda 4363$ emission line. For such a sample, which contains a large proportion of metal-rich galaxies, about $30 \%$ of the galaxies are found in the AGN region (Heckman \& Kauffmann 2003).

Because of the good spectral resolution of the SDSS spectra the singlet He I $\lambda 5015$ emission line is separated from the strong [O III] $\lambda 5007$ emission line. This feature of the 


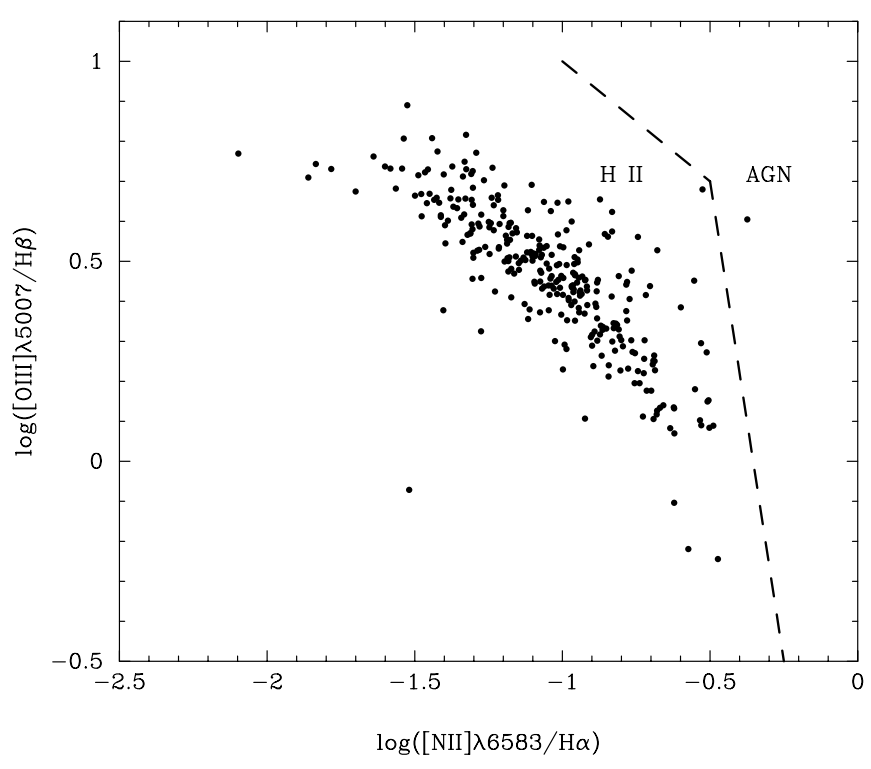

Fig. 2. Diagnostic diagram [O III] $] \lambda 5007 / \mathrm{H} \beta$ vs. $[\mathrm{N}$ II $] \lambda 6583 / \mathrm{H} \alpha$ for low-metallicity emission-line galaxies from the EDR of the SDSS. The dashed line separates the $\mathrm{H}$ II regions from galaxies ionized by a non-thermal radiation (labeled "AGN") (Osterbrock 1989).

SDSS spectra is of interest for the determination of the primordial He abundance (Cota \& Ferland 1988). The flux of the He I $\lambda 5015$ line for the case B model is $\sim 0.02$ that of $\mathrm{H} \beta$, while it is much weaker in the case $\mathrm{A}$, only $\sim 0.0005$ that of $\mathrm{H} \beta$ (Brocklehurst 1972; Aller 1984). In Fig. 3 we show the He I $\lambda 5015 / \mathrm{H} \beta$ emission line flux ratio vs. oxygen abundance $12+\log \mathrm{O} / \mathrm{H}$. The solid line is the mean value of the He I $\lambda 5015 / \mathrm{H} \beta$ emission line flux ratio, and dashed lines show $1 \sigma$ deviations. The points in Fig. 3 are scattered around a mean value of $\sim 0.02$, consistent with the case B model for He in singlet states.

In addition to the SDSS EDR, we use a sample of galaxies which has been collected primarily to study the helium abundances in low-metallicity blue compact dwarf galaxies (the HeBCD sample). This sample is gradually increasing with the addition of new objects and now includes $\sim 100$ galaxies. Most of the objects are blue compact dwarf galaxies from the First and Second Byurakan surveys, but a number of them are emission-line galaxies from the Hamburg, University of Michigan, Tololo and Case surveys. High signalto-noise ratio spectra of these galaxies in the wavelength range $13600-7400 \AA$ have been obtained with different 2 m-10 m class telescopes. Some galaxies were observed several times. We included in the sample all these independent observations. All spectra were reduced in the same way according to the prescriptions of Izotov et al. (1994, 1997a). The line fluxes corrected for extinction can be found in Izotov et al. (1994, 1996, 1997a, 1997b, 1999, 2001a, 2001b), Izotov \& Thuan (1998a, 1998b, 2003), Thuan et al. (1995, 1999), Lipovetsky et al. (1999), Guseva et al. (2000, 2001, 2003a, 2003b, 2003c), Hopp et al. (2000), Fricke et al. (2001).

The comparison of the global characteristics of the SDSS and HeBCD samples was presented by Stasińska \& Izotov (2003) and can be summarized as follows. The SDSS

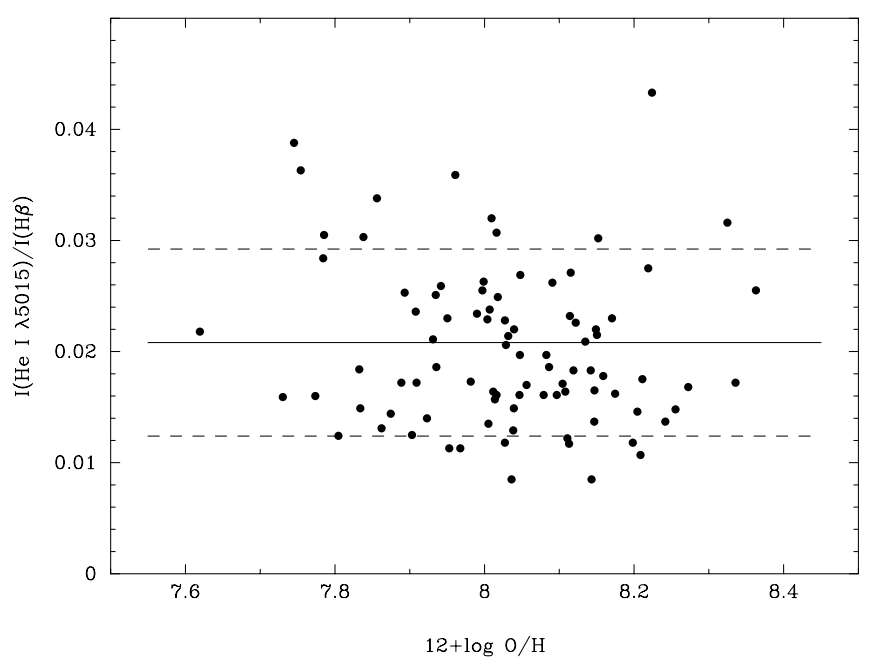

Fig. 3. He I $\lambda 5015 / \mathrm{H} \beta$ emission line flux ratio as a function of the oxygen abundance. The solid line is the mean ratio and the dashed lines are $1 \sigma$ alternatives.

galaxies are generally fainter than the HeBCD ones and their spectra are of lower signal-to-noise ratio. On average, the SDSS galaxies are more distant but their $\mathrm{H} \beta$ luminosities are similar to those of the HeBCD galaxies. The equivalent width distributions are different, being more skewed toward large equivalent widths in the HeBCD sample. Indeed, many galaxies from the HeBCD sample have been observed with the aim of deriving the primordial helium abundance, thus objects with large equivalent widths of the emission lines were preferentially selected. The distributions of $E(B-V)$ are roughly similar in both samples. For the majority of the objects, the extinction is small $(E(B-V) \lesssim 0.2)$.

\section{Element abundance determination}

The physical conditions and element abundances are derived from the emission line fluxes using the same prescriptions as in Izotov et al. (1994, 1997a). The electron temperature $T_{\mathrm{e}}\left(\mathrm{O}\right.$ III) and number density $N_{\mathrm{e}}(\mathrm{S}$ II) are derived from the [O III] $\lambda 4363 /(\lambda 4959+\lambda 5007)$ and [S II] $\lambda 6717 / \lambda 6731$ emission line flux ratios. The quality of the spectra is good enough to derive the $\mathrm{N}, \mathrm{O}, \mathrm{Ne}, \mathrm{S}$, Ar and Fe abundances. We use [N II] $\lambda 6548,6583$ emission lines for the determination of nitrogen abundance, [O II] $\lambda 3727$ or [O II] $\lambda 7320,7331$, [O III] $\lambda 4959,5007$ for the oxygen abundance, [Ne III] $\lambda 3868$ for the neon abundance, [S III] $\lambda 6312$ and [S II] $\lambda 6717,6731$ for the sulfur abundance when all these lines are detected, [Ar III] $\lambda 7135$ (and [Ar IV] $\lambda 4740$ if seen) for the argon abundance, [Fe III] $\lambda 4658,4988$ for the iron abundance. The ionization correction factors to obtain the elemental abundances from the ionic ones are the same as used by Izotov et al. (1994, 1997a). We have checked the compatibility of these ionization correction factors with those given by the photoionization models of Stasińska \& Izotov (2003) which were built to fit the observed spectral properties of the samples analyzed in the present paper. We have also run additional sequences of photoionization models, taking into account dielectronic recombination for sulfur and argon ions, and using Starburst 99 

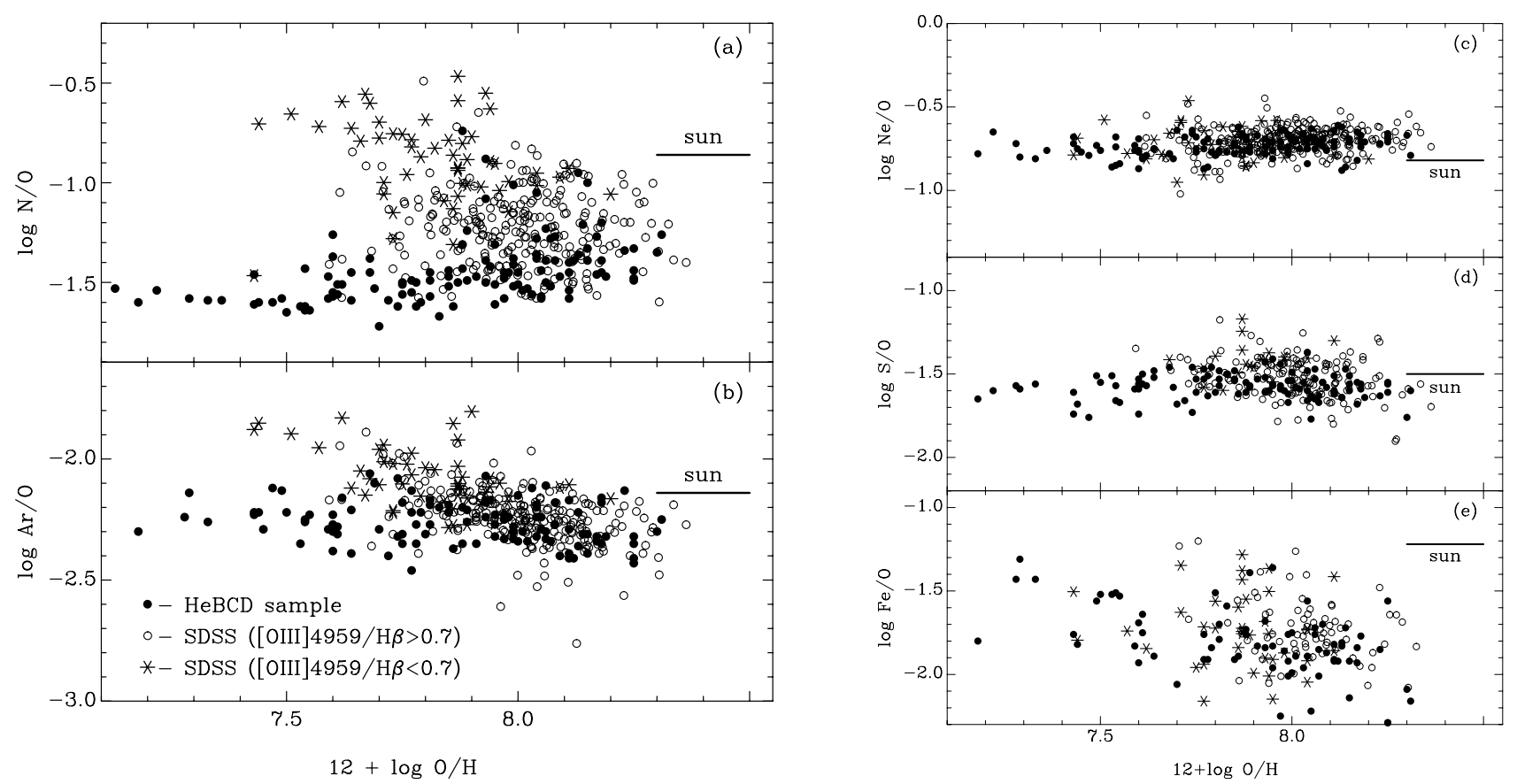

Fig. 4. $\log \mathrm{N} / \mathrm{O}$ a), $\log \mathrm{Ar} / \mathrm{O}$ b), $\log \mathrm{Ne} / \mathrm{O}$ c), $\log \mathrm{S} / \mathrm{O}$ d) and $\log \mathrm{Fe} / \mathrm{O}$ e) vs. oxygen abundance $12+\log \mathrm{O} / \mathrm{H}$ for the HeBCD and SDSS galaxies. Filled circles are the HeBCD galaxies, open circles are the SDSS galaxies with [O III] $\lambda 4959 / \mathrm{H} \beta \geq 0.7$ and asterisks are the SDSS galaxies with [O III] $\lambda 4959 / \mathrm{H} \beta<0.7$. Solar abundance ratios from Lodders (2003) are shown by solid lines.

(Leitherer et al. 1999) with the stellar model atmospheres described in Smith et al. (2002). We find that the ionization correction factors that have been used are in reasonable agreement with the predictions from the models. The only noteworthy difference is in the case of neon. To compute the abundances, it was assumed that $\mathrm{Ne} / \mathrm{O}=\mathrm{Ne}^{++} / \mathrm{O}^{++}$(which is the usual assumption). However, our models show that, as the ionization parameter decreases (or, equivalently, as the $\mathrm{O}^{++} / \mathrm{O}^{+}$ratio decreases), the proportion of $\mathrm{Ne}^{++}$with respect to $\mathrm{O}^{++}$becomes larger, since charge transfer between $\mathrm{O}^{++}$and $\mathrm{H}^{0}$ becomes more efficient to recombine $\mathrm{O}^{++}$. This effect is noticeable only when $\mathrm{O}^{++} / \mathrm{O}^{+}$is smaller than 8 , and can lead to an overestimate of $\mathrm{Ne} / \mathrm{O}$ by $0.2 \mathrm{dex}$ when $\mathrm{O}^{++} / \mathrm{O}^{+}$is equal to 1 . However, the importance of this effect also depends on the spectral energy distribution of the ionizing radiation field, which, in turn, depends on the metallicity. We have thus decided, for simplicity, to maintain the usual way to derive the $\mathrm{Ne} / \mathrm{O}$ ratio but keeping in mind any possible bias for discussion.

In Fig. 4 we show the computed N/O, Ar/O, Ne/O, S/O and $\mathrm{Fe} / \mathrm{O}$ abundance ratios vs. oxygen abundance $12+\log \mathrm{O} / \mathrm{H}$ for the SDSS and HeBCD galaxies. The HeBCD galaxies are represented by filled circles, while the SDSS galaxies are divided into galaxies with [O III] $\lambda 4959 / \mathrm{H} \beta \geq 0.7$ (represented by open circles) and galaxies with [O III] $\lambda 4959 / \mathrm{H} \beta<0.7$ (asterisks). In the latter galaxies, the equivalent width of $\mathrm{H} \beta$ is generally low and the [O III] $\lambda 4363$ emission line weak. This may cause a substantial uncertainty in the placement of the continuum due to the absorption lines of $\mathrm{H} \gamma, \mathrm{He} \mathrm{I}$ and some heavy elements blueward and redward of the [O III] $\lambda 4363$ line. Consequently, we suspect that the derived abundances may be biased. As seen in Fig. 4a, among the SDSS galaxies with [O III] $\lambda 4959 / \mathrm{H} \beta \geq 0.7$ no object was found to have $12+\log \mathrm{O} / \mathrm{H} \lesssim 7.6$. The only SDSS galaxies with such a low oxygen abundance have [O III] $\lambda 4959 / \mathrm{H} \beta \lesssim 0.7$ (labeled by asterisks). Note that all the SDSS galaxies for which $12+$ $\log \mathrm{O} / \mathrm{H} \lesssim 7.6$ have $\mathrm{N} / \mathrm{O}$ values which are several times larger than the N/O ratios derived in the most metal-deficient galaxies from the HeBCD sample. Furthermore, the asterisks show a clear trend of $\mathrm{Ar} / \mathrm{O}$ decreasing with increasing oxygen abundance (see Fig. 4b), whereas the Ar/O abundance ratio is expected to be constant over the whole range of oxygen abundance considered here (Izotov \& Thuan 1999).

This suggests that the derived oxygen abundances are underestimated, possibly due to an overestimation of the very weak [O III] $\lambda 4363$ line whose flux in these galaxies does not exceed $2 \%-4 \%$ of the $\mathrm{H} \beta$ flux. The effect of an error in the electron temperature is much weaker on the determination of the $\mathrm{Ne} / \mathrm{O}$ and $\mathrm{S} / \mathrm{O}$ abundance ratios than on the determination of the $\mathrm{N} / \mathrm{O}$ and $\mathrm{Ar} / \mathrm{O}$ ones. This explains why no trend is seen in the $\log \mathrm{Ne} / \mathrm{O}$ vs. $12+\log \mathrm{O} / \mathrm{H}$ or $\log \mathrm{S} / \mathrm{O}$ vs. $12+$ $\log \mathrm{O} / \mathrm{H}$ diagrams (from nucleosynthesis, $\mathrm{Ne} / \mathrm{O}$ and $\mathrm{S} / \mathrm{O}$ are expected to be constant, similarly to $\mathrm{Ar} / \mathrm{O}$ ). For example, if the real electron temperature in a galaxy is $10000 \mathrm{~K}$ while the [O III] $\lambda 4363 / \lambda 4959$ ratio gives an electron temperature of $20000 \mathrm{~K}$, the N/O ratio will be overestimated by a factor of about 3 if derived with [O II] $\lambda 3727$ and about 6 if derived with [O II] $\lambda 7330$, the $\mathrm{Ar} / \mathrm{O}$ ratio will be overestimated by a factor of about 2, while $\mathrm{Ne} / \mathrm{O}$ and $\mathrm{S} / \mathrm{O}$ will be underestimated by only $30 \%$. Note that such an important overestimation of the [O III] $\lambda 4363$ flux is quite likely in noisy or low excitation spectra, due to a bad placement of the continuum or due to an erroneous detection of the [O III] $\lambda 4363$ feature. However, we note that the auroral [S III] $\lambda 6312$ emission line has been detected in $30 \%$ of the spectra with [O III] $\lambda 4959 / \mathrm{H} \beta<0.7$ 

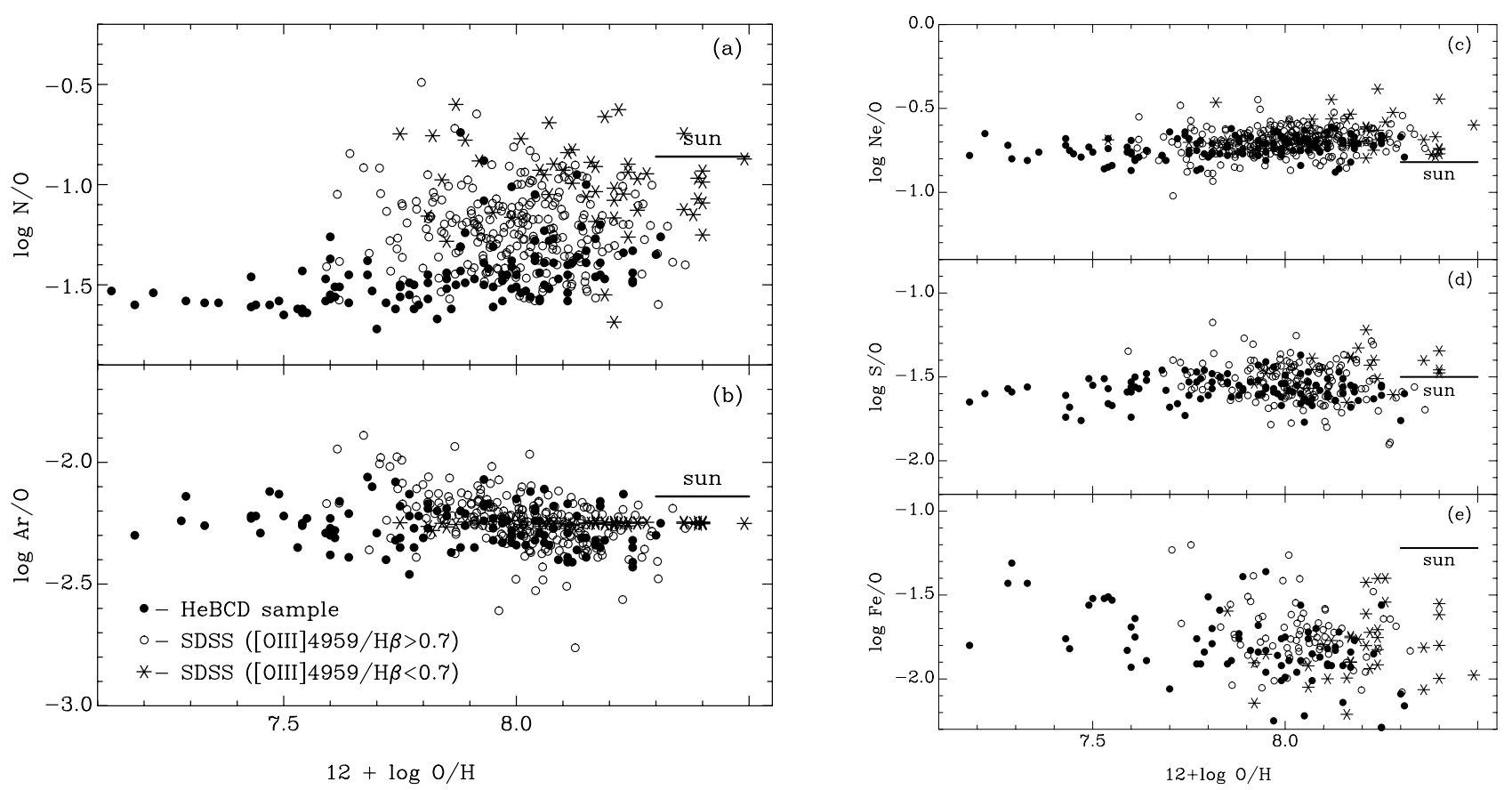

Fig. 5. Same as in Fig. 4, but after correction of the electron temperature $T_{\mathrm{e}}(\mathrm{O}$ III) in the SDSS galaxies with $[\mathrm{O}$ III $] \lambda 4959 / \mathrm{H} \beta<0.7$ (asterisks).

implying that the presence of the [O III] $\lambda 4363$ emission line is not an artefact in those spectra. It is likely that some additional heating mechanism other than the stellar radiation is important in the galaxies labeled by asterisks.

To correct the bias in the abundance determinations due to this temperature effect, we assume that all the SDSS galaxies labeled with asterisks in Fig. 4 have an $\mathrm{Ar} / \mathrm{O}$ abundance ratio equal to the mean value $(\log \mathrm{Ar} / \mathrm{O}=-2.25)$ derived by Izotov \& Thuan (1999) for the galaxies from the HeBCD sample. Then we compute the value of the electron temperature needed to achieve such a result, and use this temperature to rederive the abundances of $\mathrm{O}, \mathrm{N}, \mathrm{Ne}, \mathrm{S}$ and $\mathrm{Fe}$. The results of this procedure are shown in Fig. 5, which shows the same diagrams as Fig. 4 but with the corrected abundances. We see that, now, there is not a single SDSS galaxy with $12+\log \mathrm{O} / \mathrm{H} \lessgtr 7.6$. Also, the location of the entire sample of SDSS galaxies in the $\log \mathrm{N} / \mathrm{O}$ vs. $12+\log \mathrm{O} / \mathrm{H}$ diagram is substantially more consistent with the distribution of the galaxies from the HeBCD sample (Fig. 5a), since no galaxies with $12+\log \mathrm{O} / \mathrm{H}<7.6$ and high $\mathrm{N} / \mathrm{O}$ abundance ratio are found. On the other hand, no significant changes appear in the $\mathrm{Ne} / \mathrm{O}, \mathrm{S} / \mathrm{O}$ and $\mathrm{Fe} / \mathrm{O}$ vs. $12+$ $\log \mathrm{O} / \mathrm{H}$ diagrams except that the galaxies represented by asterisks are shifted towards higher oxygen abundance with respect to Fig. 4. This supports the correctness of our approach.

\section{Discussion}

The distribution of the SDSS galaxies from the Early Data Release (open circles and asterisks) in the different panels of Fig. 5 confirms previous findings for HeBCD galaxies (Thuan et al. 1995; Izotov \& Thuan 1999). The only striking difference is that no extremely metal-deficient galaxies with $12+\log \mathrm{O} / \mathrm{H}<7.6\left(Z<Z_{\odot} / 12\right)$ are found in the SDSS sample. Recall however that we have excluded from consideration galaxies with strong but clipped emission lines. Some of those galaxies could actually be metal-poor. Meanwhile, the SDSS Data Release 1 (DR1) has become available ${ }^{2}$. Galaxies from the EDR have re-reduced spectra included in the DR1. Among galaxies from the DR1 sample only a few have spectra with clipped emission lines. Therefore, using the data from the DR1 sample, we have checked whether very metal-poor galaxies are present in the EDR, among those which have been excluded from our consideration because of clipped lines. No such galaxies were found. We suspect that the selection criteria used in the SDSS to extract galaxies from multi-colour imaging data for follow-up spectroscopic work (Stoughton et al. 2002) are biased against the most metal-poor galaxies. First, in the SDSS galaxies are distinguished from stars by morphology. But distant blue compact dwarf galaxies can be morphologically indistinguishable from stars, and some of them might even appear in the quasar sample of the SDSS. In fact, we find several cases when low-metallicity emission-line galaxies were classified as QSOs. Second, extremely metal-poor galaxies are expected to be of very low luminosity because of the metallicity-luminosity relation (e.g., Skillman et al. 2003) and for that reason they are rejected from the list of targets for SDSS spectroscopy. The SDSS aims at obtaining a complete sample of galaxies brighter than a Petrosian magnitude $r_{*}=17.77$ (see Stoughton et al. 2002). Galaxies with an absolute brightness of $\sim-14 \mathrm{mag}$, similar to that of I $\mathrm{Zw} 18$, are already fainter than the completeness limit at a redshift of 0.01 . The selection criteria for the HeBCD galaxies were completely different. The objects were chosen from objective prism surveys to have large equivalent widths of the $\mathrm{H} \beta$

\footnotetext{
${ }^{2}$ http://www.sdss.org/dr1
} 

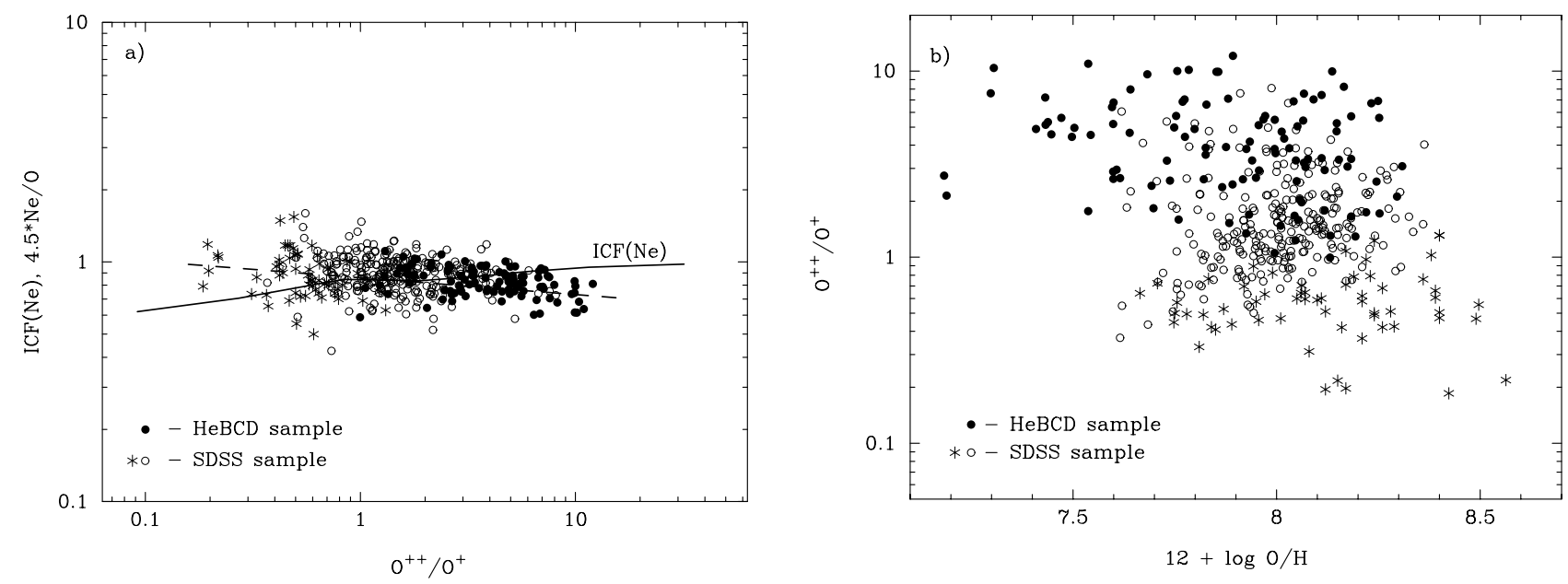

Fig. 6. a) The $\mathrm{Ne} / \mathrm{O}$ abundance ratios for our sample galaxies as a function of $\mathrm{O}^{++} / \mathrm{O}^{+}$ratio (same symbols as Fig. 4). The dashed line shows the trend of the data derived from the observations. The solid line shows the ionization correction factor $I C F(\mathrm{Ne})$ as derived from photoionization models with metallicity $Z=Z_{\odot} / 5$ (see text). b) The observed values of $\mathrm{O}^{++} / \mathrm{O}^{+}$as a function of $12+\log \mathrm{O} / \mathrm{H}$.

and [O III] $\lambda 4959,5007$ emission lines, mainly for the determination of the pregalactic helium abundance. Therefore the metallicity distributions of the two samples are expected to be different.

We find no significant trends with the oxygen abundance for the $\mathrm{Ne} / \mathrm{O}$ and $\mathrm{S} / \mathrm{O}$ abundance ratios (Figs. 5c, 5d), although higher-metallicity galaxies have $\log \mathrm{Ne} / \mathrm{O}$ by $\sim 0.1 \mathrm{dex}$ higher than low-metallicity galaxies. This small trend is likely caused by a bias in the $\mathrm{Ne} / \mathrm{O}$ abundance determination, as discussed before. Formally, one can write $\mathrm{Ne} / \mathrm{O}=$ $I C F(\mathrm{Ne}) \times\left(\mathrm{Ne}^{++} / \mathrm{O}^{++}\right)$. As explained in Sect. 3, we have adopted $I C F(\mathrm{Ne})=1$ whereas it is smaller than unity and decreases with decreasing of $\mathrm{O}^{++} / \mathrm{O}^{+}$. In Fig. $6 \mathrm{a}$, we show with the solid curve the value of $I C F(\mathrm{Ne})$ as a function of $\mathrm{O}^{++} / \mathrm{O}^{+}$ for a sequence of photoionization models with metallicity $Z=$ $Z_{\odot} / 5$, computed using the stellar atmospheres from Smith et al. (2002), but otherwise identical to the sequence of models that matches the "high metallicity bin" of our sample galaxies (cf. Stasińska \& Izotov 2003 for more details). We see that indeed $I C F(\mathrm{Ne})$ is slightly smaller than unity at low excitation. The effect on $I C F(\mathrm{Ne})$ is even larger for lower metallicity, because the stellar energy distribution in the Lyman continuum is then harder. However, galaxies with lower metallicities tend to have higher $\mathrm{O}^{++} / \mathrm{O}^{+}$, as seen in Fig. 6b, therefore the bias in the derived $\mathrm{Ne} / \mathrm{O}$ ratio ends up being unimportant. In Fig. 6a, we have also plotted the derived $\mathrm{Ne} / \mathrm{O}$ values for our sample galaxies, and drawn as a dashed line the observed trend, which is clearly opposite to the trend in $I C F(\mathrm{Ne})$. The correction for $I C F(\mathrm{Ne})$ shown in Fig. 6a would thus reduce this trend.

We find that the $\mathrm{Fe} / \mathrm{O}$ abundance ratios are generally significantly smaller than solar and similar to the ones derived in the most metal-poor stars in the Galaxy (Caretta et al. 2000). This may argue in favour either of a moderate depletion of iron onto dust grains or of production of iron being dominated by type II supernovae (arising from massive stars). However we note a slight decrease of the $\mathrm{Fe} / \mathrm{O}$ abundance ratio with increasing metallicity (by $\sim 0.2$ dex when the oxygen abundance increases by one order of magnitude as seen in Fig. 5e).
This implies that some depletion occurs in the high-metallicity galaxies. Nevertheless, the similarity of the Fe/O abundance ratios in the studied galaxies and halo stars favours the production of iron being dominated by type II supernovae, which implies an age of $<1-2$ Gyr, i.e. before the contribution of type Ia supernovae (which arise from intermediate mass stars) becomes important (Thuan et al. 1995; Izotov \& Thuan 1999).

We now compare the abundance patterns we find for the local metal-poor emission-line galaxies with those found for DLAs (e.g., Centurión et al. 2003). Many DLAs actually follow the N/O vs. O/H distribution of the local dwarf emissionline galaxies. This suggests similar star formation histories and evolutionary status for these two classes of objects. However, we have found no local counterparts of the high-redshift DLAs with $\log \mathrm{N} / \mathrm{O} \lesssim-1.6$. It has been proposed (e.g., Centurión et al. 2003) that DLAs with low N/O abundance ratio are young systems just starting to form stars and where both $\mathrm{N}$ and $\mathrm{O}$ are produced by massive stars, while DLAs with high $\mathrm{N} / \mathrm{O}$ abundance ratio as well as local emission-line galaxies are older, with an age of at least several hundred Myr, to allow the intermediate-mass stars to deliver their nitrogen. This conclusion is in line with predictions of the evolution of rotating stars (Meynet \& Maeder 2002). However, such an interpretation presents problems. In the $\mathrm{N} / \mathrm{O}$ vs. $\mathrm{O} / \mathrm{H}$ plane, DLAs with high $\mathrm{N} / \mathrm{O}$ and DLAs with low $\mathrm{N} / \mathrm{O}$ abundance ratios form two discrete groups. It is not clear, as discussed by Centurión et al. (2003), why only very young DLAs with low N/O and relatively evolved DLAs with high N/O are found, while no intermediate case is known. New observations and studies of a larger sample of DLAs and local emission-line galaxies are necessary to clarify the situation.

If the nitrogen and oxygen production scenario discussed above holds, then the low N/O dispersion in the galaxies with low oxygen abundances in Fig. 5a can be explained by massive stars continuously producing oxygen and intermediate-mass stars continuously producing primary nitrogen. However, the large spread of the N/O abundance ratios at higher metallicities needs to be explained. In Fig. 7 we show the N/O abundance 


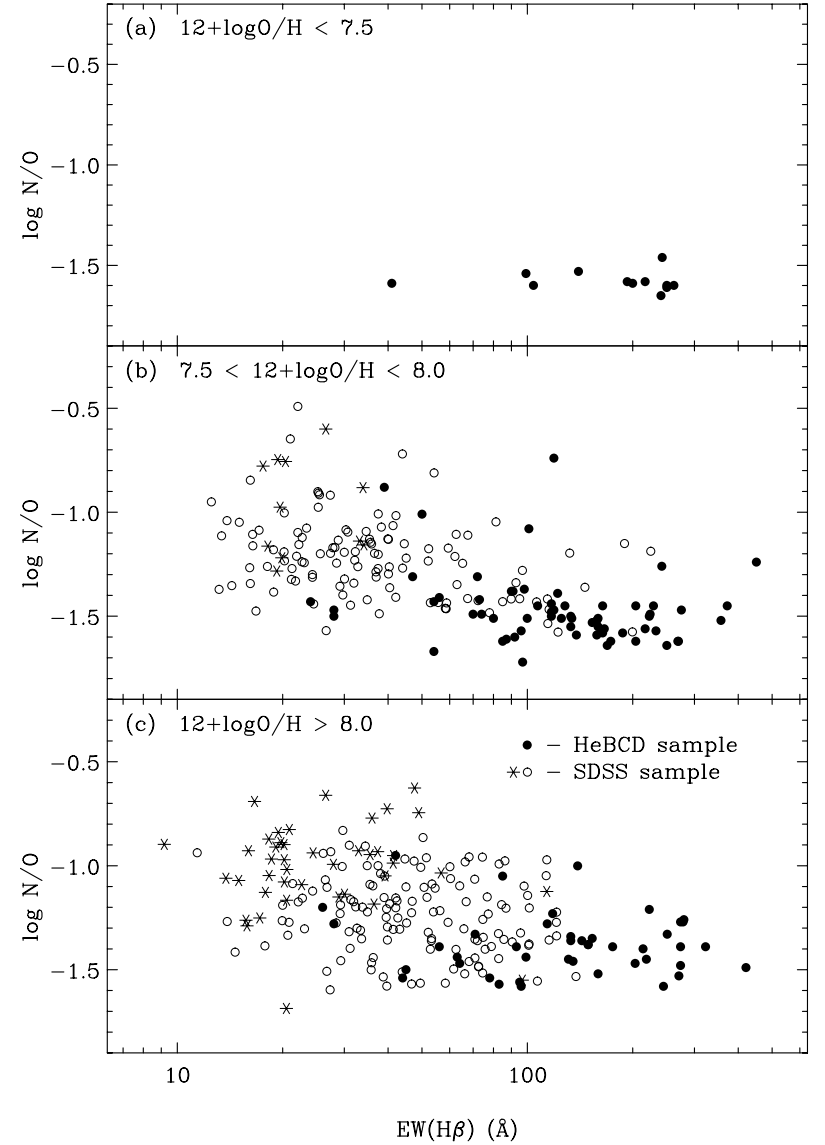

Fig. 7. Nitrogen-to-oxygen abundance ratio, $\log \mathrm{N} / \mathrm{O}$, vs. equivalent width of $\mathrm{H} \beta$ emission line, $E W(\mathrm{H} \beta)$, for the galaxies in three metallicity bins defined as in Stasińska \& Izotov (2003).

ratios as a function of the $\mathrm{H} \beta$ equivalent width $E W(\mathrm{H} \beta)$. The samples are split into three metallicity bins following Stasińska \& Izotov (2003). No trend is seen in the low-metallicity bin (but the number of the galaxies and the range of $E W(\mathrm{H} \beta)$ are small). On the other hand, clear trends are present for the galaxies in the intermediate- and high-metallicity bins for which the $\mathrm{N} / \mathrm{O}$ abundance ratio and its dispersion increase when $E W(\mathrm{H} \beta)$ decreases. Since $E W(\mathrm{H} \beta)$ is an indicator of the age of the latest burst of star formation in the galaxy (but see the discussion by Stasińska \& Izotov 2003), the trends and large dispersions of N/O in the intermediate- and high-metallicity bins (Fig. 7) can be explained by the additional production of nitrogen in massive Wolf-Rayet stars arising from the latest burst, on top of the nitrogen produced by intermediate-mass stars. This effect is expected to be lower for the $\mathrm{H}$ II regions in the low-metallicity bin because of a significantly lower fraction of Wolf-Rayet stars at low metallicities. Note, however, that a few cases of extremely low-metallicity galaxies have been reported in the literature with $\log \mathrm{N} / \mathrm{O}$ slightly larger than -1.6 (van Zee 2000; Skillman et al. 2003). These objects have $E W(\mathrm{H} \beta)$ of the order of $100 \AA$, therefore the most massive stars from the latest burst have probably had time to evolve and enrich the nebula in nitrogen. A larger sample of extremely low-metallicity galaxies would be welcome to study this issue in more detail.

\section{Conclusions}

We have discussed in this study the abundances of the heavy elements in the 310 emission-line galaxies from the sample of the Sloan Digital Sky Survey (SDSS) Early Data Release (EDR) with a detected [O III] $\lambda 4363$ emission line, allowing a direct element abundance determination based on the electron temperature. We have shown that the abundances are biased for galaxies with spectra of low excitation, and we attribute this bias mainly to a false detection of [O III] $\lambda 4363$ or to a wrong placement of the continuum due to a low signal-to-noise ratio and adjacent stellar absorption lines. However, we cannot exclude that an additional heating mechanism different from the stellar radiation may be important for low-excitation galaxies. We have corrected for this bias by requiring that the $\mathrm{Ar} / \mathrm{O}$ ratio be equal to the expected value. After correcting for this bias and merging the SDSS sample with the sample of the galaxies with large equivalent widths of emission lines (HeBCD sample), we obtain a sample of $\sim 400$ emission-line galaxies in total. Our main conclusions are as follows:

1. Despite an examination of the entire SDSS EDR sample of galaxies, we found no galaxy with extremely low metallicity $\left(12+\log \mathrm{O} / \mathrm{H}<7.6\right.$, i.e. $\left.Z<Z_{\odot} / 12\right)$ while the HeBCD sample which consists of $\sim 100$ galaxies contains about 15 such galaxies. We suspect that the spectroscopic data base of the SDSS actually discriminates against the most metal-poor galaxies.

2. Emission-line galaxies from the SDSS sample in general follow the distributions of the N/O, Ne/O, S/O, Ar/O, $\mathrm{Fe} / \mathrm{O}$ abundance ratios vs. oxygen abundance found earlier for the galaxies in the HeBCD sample (Thuan et al. 1995; Izotov \& Thuan 1999).

3. The $\alpha$ element-to-oxygen abundance ratios do not show any significant trend with oxygen abundance.

4. The Fe/O abundance ratio shows a significant underabundance of iron relative to oxygen as compared to solar, suggesting either depletion of iron onto dust grains or a dominant production of iron in the massive supernovae of the type II. We favour the latter explanation, which implies an age of less than $\sim 1-2$ Gyr.

5. No galaxy with $\log \mathrm{N} / \mathrm{O} \lesssim-1.6$ was found suggesting that local low-metallicity emission-line galaxies are different from those as high-redshift DLAs with the considerably lower $\log \mathrm{N} / \mathrm{O}$ of $\sim-2.3$. These DLAs are considered to be truly young galaxies, with nitrogen produced only by massive stars. If this interpretation is correct, then our sample of $\sim 400$ dwarf emission-line galaxies contains no extremely young galaxy (i.e. with an age $\$ 100-300 \mathrm{Myr}$ ).

6. Our data indicate a gradual enrichment of the galaxies in nitrogen by massive stars from the most recent starburst. The effect is best seen among galaxies from our sample that have intermediate metallicities.

Acknowledgements. Y.I.I. acknowledges the support of the University of Paris 7 and the Observatoire de Paris, where part of this work was carried out. Y.I.I. and N.G.G. acknowledge the support of the Swiss SCOPE 7UKPJ62178 grant. T.X.T. and Y.I.I. acknowledge the partial financial support of NSF grant AST 02-05785. All the authors acknowledge the work of the Sloan Digital Sky Survey (SDSS) team. 
The SDSS is a joint project of The University of Chicago, Fermilab, the Institute for Advanced Study, the Japan Participation Group, the Johns Hopkins University, the Los Alamos National Laboratory, the Max-Planck-Institute for Astronomy (MPIA), the Max-PlanckInstitute for Astrophysics (MPA), New Mexico State University, Princeton University, the United States Naval Observatory, and the University of Washington. Funding for the project has been provided by the Alfred P. Sloan Foundation, the Participating Institutions, the National Aeronautics and Space Administration, the National Science Foundation, the U.S. Department of Energy, the Japanese Monbukagakusho, and the Max Planck Society.

\section{References}

Aller, L. H. 1984, Physics of Thermal Gaseous Nebulae (Dordrecht: Reidel)

Brocklehurst, M. 1972, MNRAS, 157, 211

Caretta, E., Gratton, R. G., \& Sneden, C. 2000, A\&A, 356, 238

Centurión, M., Molaro, P., Vladilo, G., et al. 2003, A\&A, 403, 55

Cota, S. A., \& Ferland, G. J. 1988, ApJ, 326, 889

Fricke, K. J., Izotov, Y. I., Papaderos, P., Guseva, N. G., \& Thuan, T. X. 2001, AJ, 121, 66

Garnett, D. R. 1990, ApJ, 363, 142

Guseva, N. G., Izotov, Y. I., \& Thuan, T. X. 2000, ApJ, 531, 776

Guseva, N. G., Izotov, Y. I., Papaderos, P., et al. 2001, A\&A, 378, 756

Guseva, N. G., Papaderos, P., Izotov, Y. I., et al. 2003a, A\&A, 407, 75

Guseva, N. G., Papaderos, P., Izotov, Y. I., et al. 2003b, A\&A, 407, 91

Guseva, N. G., Papaderos, P., Izotov, Y. I., et al. 2003c, A\&A, 407, 105

Heckman, T. M., \& Kauffmann, G. 2003, in Star Formation through Time, Granada, Sep. 2002, ASP, ed. E. Perez, R. Gonzalez Delgado, \& G. Tenorio Tagle, in press

Henry, R. B. C., Edmunds, M. G., \& Köppen, J. 2000, ApJ, 541, 660
Hopp, U., Engels, D., Green, R. F., et al. 2000, A\&AS, 142, 417

Hunt, L. K., Thuan, T. X., \& Izotov, Y. I. 2003, ApJ, 588, 281

Izotov, Y. I., \& Thuan, T. X. 1998, ApJ, 497, 227

Izotov, Y. I., \& Thuan, T. X. 1998, ApJ, 500, 188

Izotov, Y. I., \& Thuan, T. X. 1999, ApJ, 511, 639

Izotov, Y. I., \& Thuan, T. X. 2003, ApJ, in press, preprint [astro-ph/0310421]

Izotov, Y. I., Thuan, T. X., \& Lipovetsky, V. A. 1994, ApJ, 435, 647

Izotov, Y. I., Dyak, A. B., Chaffee, F. H., Foltz, C. B., Kniazev, A. Y., \& Lipovetsky, V. A. 1996, ApJ, 458, 524

Izotov, Y. I., Thuan, T. X., \& Lipovetsky, V. A. 1997a, ApJS, 108, 1

Izotov, Y. I., Lipovetsky, V. A., Chaffee, F. H., et al. 1997, ApJ, 476, 698

Izotov, Y. I., Chaffee, F. H., Foltz, C. B., et al. 1999, ApJ, 527, 757

Izotov, Y. I., Chaffee, F. H., \& Green, R. F. 2001a, ApJ, 562, 727

Izotov, Y. I., Chaffee, F. H., \& Schaerer, D. 2001b, A\&A, 378, L45

Leitherer, C., Schaerer, D., Goldader, J. D., et al. 1999, ApJS, 123, 3

Lipovetsky, V. A., Chaffee, F. H., Izotov, Y. I., et al. 1999, ApJ, 519, 177

Lodders, K. 2003, ApJ, 591, 1220

Lu, L., Sargent, W. L. W., \& Barlow, T. A. 1998, AJ, 115

Maeder, A. 1992, A\&A, 264, 105

Meynet, G., \& Maeder, A. 2002, A\&A, 381, L25

Osterbrock, D. E. 1989, Astrophysics of gaseous nebulae and active galactic nuclei (Mill Valley: University Science Books)

Sargent, W. L. W., \& Searle, L. 1970, ApJ, 162, L155

Skillman, E., Côté, S., \& Miller, B. W. 2003, AJ, 125, 610

Smith, L. J., Norris, R. P. F., \& Crowther, P. A. 2002, MNRAS, 337, 1309

Stasińska, G., \& Izotov, Y. I. 2003, A\&A, 397, 71

Stoughton, C., Lupton, R. H., Bernardi, M., et al. 2002, AJ, 123, 485

Thuan, T. X., Izotov, Y. I., \& Lipovetsky, V. A. 1995, ApJ, 445, 108

Thuan, T. X., Izotov, Y. I., \& Foltz, C. B. 1999, ApJ, 525, 105

van Zee, L. 2000, ApJ, 543, L31 